\title{
The Role of Internal Models in Motion Planning and Control: Evidence from Grip Force Adjustments during Movements of Hand-Held Loads
}

\author{
J. Randall Flanagan ${ }^{1}$ and Alan M. Wing ${ }^{2}$ \\ ${ }^{1}$ Department of Psychology, Queen's University, Kingston, Ontario, Canada K7L 3N6, and ${ }^{2}$ Medical Research Council \\ Applied Psychology Unit, Cambridge CB2 2EF, United Kingdom
}

We investigated the issue of whether or not the CNS makes use of an internal model of the motor apparatus in planning and controlling arm movements. In particular, we tested the ability of subjects to predict different hand-held loads by examining grip force adjustments used to stabilize the load in the hand during arm movements.

Subjects grasped a manipulandum using a precision grip with the tips of the thumb and index finger on either side. The grip force (normal to the contact surfaces) and the load force (tangential to the surfaces) were measured, along with the trajectory of the hand. The manipulandum was attached to two servo-controlled linear motors used to create inertial and viscous loads as well as a composite load, including inertial, viscous, and elastic components.
The form of the hand trajectory was independent of load for some subjects but varied systematically across load conditions in others. Nevertheless, under all load conditions and in all subjects, grip force was modulated in parallel with, and thus anticipated, fluctuations in load force despite the marked variation in the form of the load function. This indicates that the CNS is able to predict the load force and the kinematics of hand movement on which the load depends. We suggest this prediction is based on an internal model of the motor apparatus and external load and is used to determine the grip forces required to stabilize the load.

Key words: internal model; feedforward control; reaching movement; dynamics; grip force; load force; hand trajectory
A current controversy in motor control is whether the CNS makes use of an internal model of the motor apparatus in planning and executing goal-directed movements. A number of investigators have suggested that an internal model is used either to predict the movement consequences of motor commands (forward model) (Jordan and Rumelhart, 1992; Miall et al., 1993; Jordan et al., 1994; Wolpert et al., 1995) or to determine the commands needed to achieve a desired movement trajectory (inverse model) (Saltzman, 1979; Atkeson, 1989; Uno et al., 1989; Hollerbach, 1990). However, other workers have proposed control theories that explicitly reject the notion of an internal model (Bizzi et al., 1984; Flash, 1987; Bullock and Grossberg, 1988; Feldman et al., 1990; Flanagan et al., 1993a). We tested the hypothesis that an internal model is used to predict movement-dependent loads by examining grip force adjustments during arm movements with hand-held loads. In particular, we investigated whether changes in grip force anticipate, or predict, fluctuations in load force under different load conditions.

When an object is held with the tips of the thumb and index finger at the sides, grip (or normal) force allows the development of frictional force to counteract the load (or tangential) force. In a series of elegant studies, Johansson and colleagues (Johansson and Westling, 1984; Johansson et al., 1992) have shown that, when lifting objects or pulling on fixed loads, grip force (GF) is adjusted

\footnotetext{
Received Oct. 31, 1996; accepted Dec. 6, 1996.

This work was supported by the Natural Sciences and Engineering Council of Canada, the British Medical Research Council, and a Wellcome Research Travel Grant to J.R.F.

Correspondence should be addressed to Dr. J. Randall Flanagan at the above address.

Copyright (C) 1997 Society for Neuroscience 0270-6474/97/171519-10\$05.00/0
}

in parallel with changes in load force (LF) such that it is always slightly greater than the minimum required to prevent slip.

Recently, we have examined GF adjustments during rapid arm movements with hand-held objects. We have shown that GF is modulated in parallel with fluctuations in the accelerationdependent inertial load (Flanagan et al., 1993b; Flanagan and Wing, 1993, 1995; Flanagan and Tresilian, 1994). This finding indicates that the CNS is able to predict precisely the movementinduced load, and it seems reasonable to suggest that this prediction is based on an internal model of the motor apparatus and external load.

However, to date we have examined only inertial loads and cannot rule out the possibility that the precise anticipatory modulation of GF with fluctuations in LF is restricted to these loads, perhaps reflecting a relatively fixed relation between motor commands for arm movement to those governing GF. The relation between arm movement motor commands and the load experienced at the hand will depend on the type of load being moved. Thus, to adjust GF precisely for fluctuations in LF under different load conditions, it is necessary to alter the mapping between arm movement and GF commands. In this paper, we examine the coordination of GF and LF during movements of inertial, viscous, and elastic loads. Should GF and LF be coupled precisely under all three load conditions, we would have evidence that the commands for grip force are not rigidly linked to the commands for arm movement but, instead, are based on an internal model of the motor apparatus and external load.

\section{MATERIALS AND METHODS}

Subjects. Fifteen subjects, including members of the subject panel and staff of the MRC Applied Psychology Unit, participated in this study with 
A Servo-Controlled Linear Motors

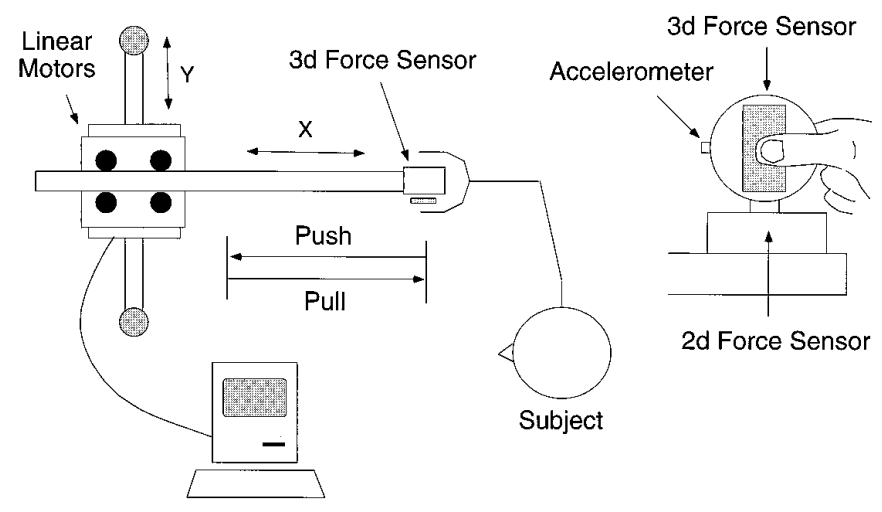

Figure 1. A, Top view of the experimental setup. Subjects grasp a manipulandum attached to two force-served linear motors mounted at right angles to give motion in the horizontal plane. $B$, Side view of the manipulandum instrumented with force sensors to measure grip force normal to the contact surface and load forces tangential to the surface.

informed consent. The panel subjects were paid for their participation. The subjects included eight men and seven women between the ages of 24 and 40 . Ten of the subjects completed all three experiments described below and, thus, the present analyses will focus on their data.

Experimental setup. Subjects grasped a manipulandum instrumented with a three-dimensional force transducer (Novatech, model F233) that recorded the grip force, normal to the grip surface, and the horizontal and vertical load forces tangential to the surface. A one-dimensional accelerometer (Entran, model EGB-125-10D) was taped to the manipulandum to measure acceleration in the direction of movement. The manipulandum was attached to a two-dimensional force transducer (Novatech, model F232) that recorded forces in the horizontal plane. The two-dimensional transducer was fixed to two force-served linear motors coupled at right angles to give motion in the horizontal plane (see Fig. 1). Position was provided by an optical encoder attached to the motor.

A Macintosh IIfx computer with a 16 bit A-D board (National Instruments, model NBMI016X) was used to sample the position and acceleration of the manipulandum and the five forces measured by the twodimensional and three-dimensional force transducers at $500 \mathrm{~Hz}$. The computer also was used to control the load force, resisting the movement on the basis of the acceleration, velocity (integrated from acceleration), or position of the manipulandum. The force servo rate (i.e., the speed of the control loop) was $80 \mathrm{~Hz}$.

In the inertial and viscous load conditions, we were able to generate nearly pure inertial and viscous loads. However, we were unable to achieve a pure elastic load and thus will refer to this load as a composite load to reflect the fact that it also contained inertial and viscous components. Regression analysis, in which the measured load force was regressed against position, velocity, and acceleration, was used to estimate the mass $(m)$, viscosity $(b)$, and elasticity $(k)$ for each trial. Then these estimates were averaged across trials to obtain single estimates for each of the 10 subjects; the latter then were averaged across subjects to obtain a single estimate for each load condition. In the inertial condition, the load was primarily inertial $(m=1.41 \mathrm{~kg})$, but there was a small viscous component $(b=2.55 \mathrm{~kg} / \mathrm{sec})$. In the viscous condition, the load was predominantly viscous $(b=18.80 \mathrm{~kg} / \mathrm{sec})$, but there was a small inertial component $(m=0.70 \mathrm{~kg})$. In the complex load condition, there was a large elastic component $(k=31.32 \mathrm{~N} / \mathrm{m})$ as well as substantial inertial $(m=1.09 \mathrm{~kg})$ and viscous $(b=7.84 \mathrm{~kg} / \mathrm{sec})$ components. Matchedsamples $t$ tests (using subject means) revealed that there were no significant differences in these estimates as a function of movement direction (push vs pull; see below). It is important to note that the fact that we used a composite load rather than a pure elastic load has no bearing on the logic of the experiment. We simply wished to generate three markedly different loads to assess whether the coupling between GF and LF is independent of the type of load.

Experimental procedure. Each subject performed 40 trials in each of the three load conditions. All subjects first completed the inertial load trials, followed by the viscous and then the composite load trials. Each set of 40 trials consisted of alternating movements away from (pushes) and toward (pulls) the body, moving the manipulandum a distance of $26 \mathrm{~cm}$ between movement end points (marked with strips of bright tape). Subjects were instructed to make each movement a single action and were told not to be too concerned about the accuracy of end point positioning. The subjects also were asked to move the manipulandum in a straight line. Because the manipulandum was free to move laterally (the lateral load force was servo-controlled to zero), the subjects received visual feedback if they erroneously pushed the manipulandum sideways. All subjects were able to produce straight line movements consistently. Because of the position of the subject in relation to the apparatus (see Fig. 1), movements involved shoulder and elbow rotations with relatively little wrist motion. However, no specific instructions regarding joint motions were provided to the subject.

Data analysis. The raw force and kinematic data were smoothed digitally with a Butterworth fourth-order, zero-phase lag, low-pass filter. A cutoff frequency of $6 \mathrm{~Hz}$ was used for the position signal; a cutoff of $8 \mathrm{~Hz}$ was used for remaining signals. Velocity was obtained by digitally integrating the acceleration signal. The LF was computed as the resultant of the horizontal and vertical forces (measured from the three-dimensional force transducer) tangential to the grasp surface. The ratio of GF to LF also was computed and will be referred to as the force ratio. The start time of the movement was defined as the point at which the absolute acceleration of the manipulandum first exceeded $10 \mathrm{~cm} / \mathrm{sec}^{2}$, and the end time was defined as the point at which the absolute acceleration dropped below $10 \mathrm{~cm} / \mathrm{sec}^{2}$ for the last time. ANOVA and linear regression analysis were used to test various experimental effects. A $p$ value of 0.05 was considered statistically significant.

\section{RESULTS}

The results consist of two main sections. The first focuses on the coordination of grip force (GF) and load force (LF) under the three load conditions. In the second section, the form of the hand velocity profiles is considered. In this paper, we have focused on the kinematic and kinetic patterns observed during steady-state performance after subjects completed initial trials under each load condition. A report examining the performance during the initial trials under the different load conditions is forthcoming.

\section{Coordination of grip force and load force}

The main result of this paper is that, in each load condition, GF is adapted to the LF such that the two forces fluctuate in parallel. This finding is illustrated in Figure 2, which shows kinematic and kinetic records from a single push trial under each of the three load conditions for one subject (S5). In each panel the shaded trace in the top part of the figure indicates the (primary) kinematic variable determining the component of load force in the direction of movement $(H F)$. Under the inertial load, GF has two peaks that correspond in time to the peaks in LF. Moreover, GF tracks LF throughout the movement. The initial rise in GF allows the hand to accelerate the object without slip; the second GF rise permits the hand to decelerate the object without slip. Under the viscous load, the GF function exhibits a single peak that coincides with the peak LF. The GF and LF peaks occur near the middle of the movement when acceleration is close to zero and velocity is close to its maximum. Thus, peak GF occurs at a different point in the movement than either of the GF peaks observed under the inertial load. Finally, in the composite load condition, GF again is modulated in parallel with the LF, which primarily reflects changes in position but also exhibits some covariation with acceleration and velocity.

In Figures 3-5 we present superimposed traces for pulls and pushes under each load condition for two subjects (S8, S10). These traces were selected from the last 10 push and last 10 pull trials, by which time the subjects had adapted to the load. The panels in these figures show kinematics (above) and kinetics (below) as well as the ratio of GF to LF, or force ratio. Under the 


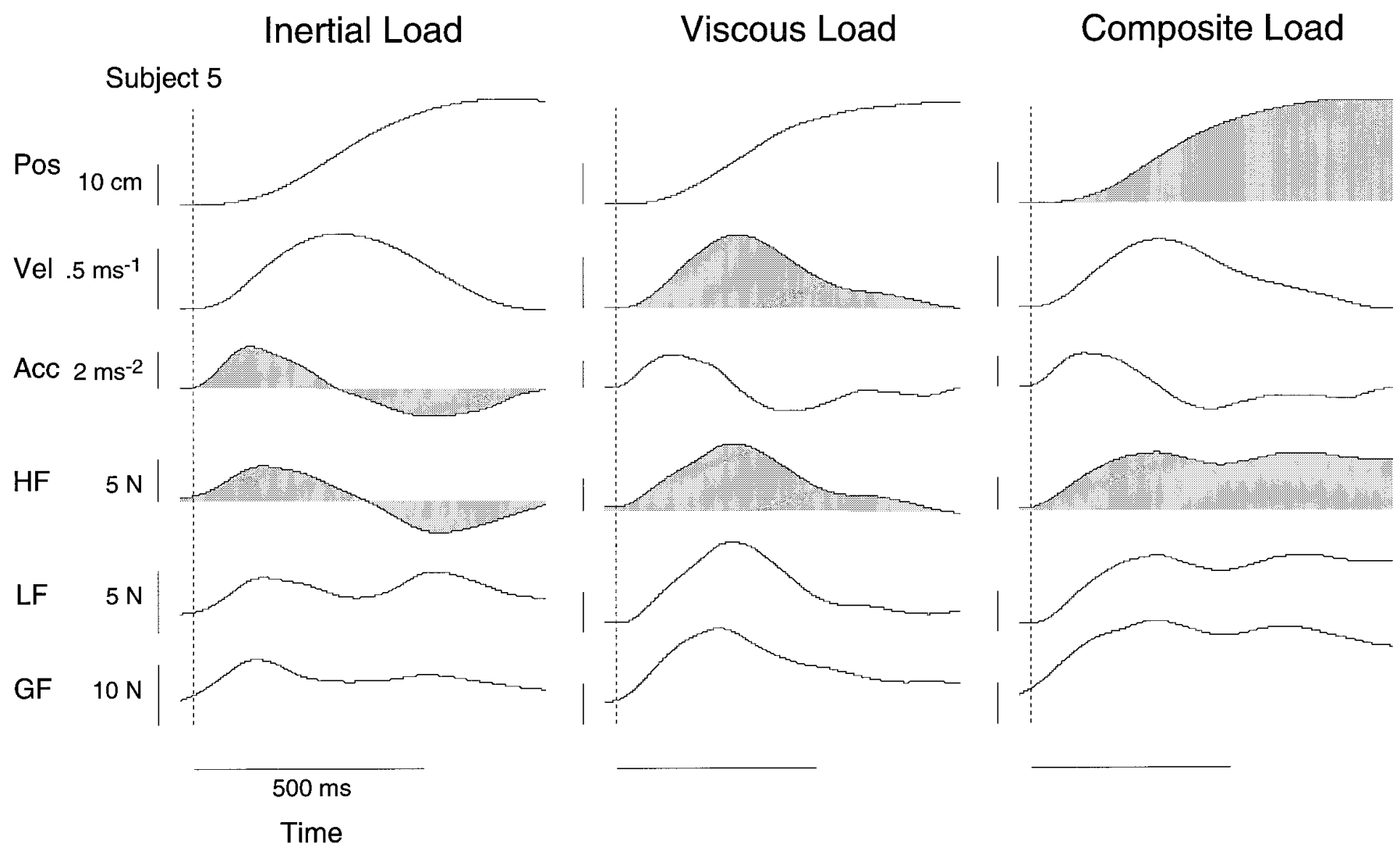

Figure 2. Single kinematic and force records from one subject under the three load conditions. Shaded regions indicate the horizontal load force (HF) resisting the movement and the primary kinematic variable on which this component of the load depended. Under all three load conditions, grip force $(G F)$ is adjusted in parallel with fluctuations in load force $(L F)$, the resultant load tangential to the grasp surface. All calibration bars start at zero. Dashed vertical lines indicate movement onset.

inertial load condition, all subjects exhibited velocity profiles that were unimodal and approximately symmetric. Similar velocity profiles have been described previously for movements under inertial loading (Ruitenbeek, 1984; Atkeson and Hollerbach, 1985; Stein et al., 1988; Bock, 1990). In the viscous and composite load conditions, the form of the velocity profile varied across subjects. Approximately one-half of the subjects, including S8, exhibited nearly symmetric velocity profiles, regardless of the load. However, other subjects, S10 among them, exhibited positively skewed velocity profiles under the viscous and/or complex load conditions. One subject, S6, produced velocity profiles that were skewed negatively in the viscous condition.

As illustrated in Figures 3-5, the form of the GF function varies systematically across the three load conditions. However, under all load conditions, GF fluctuates in parallel with LF. Right from the beginning of the movement, GF increases and decreases in phase with LF. If GF were adjusted in reaction to changes in LF, we would expect modulations in GF to lag behind changes in LF. The fact that the two forces vary in phase indicates that GF anticipates LF. We have previously reported parallel changes in GF and LF during vertical arm movements with hand-held objects in which the load acting on the object included gravitational and acceleration-dependent inertial components (Flanagan and Wing, 1993, 1995). The present results indicate that the tight coupling between GF and LF extends to other loads. Because LF depends on both the trajectory of the hand and the load properties, the precise anticipatory GF adjustments evident in Figures 3-5 indi- cate that the motor system is able to predict both the trajectory and the load.

To assess the phase relation between GF and LF quantitatively, we computed cross-correlations between GF rate and LF rate, the first time derivatives of GF and LF. (Force rates were used to obtain a more sensitive measure of the phase relation.) The phase lag at which the maximum correlation occurred was computed for each of the last 20 trials from each load condition. For each subject we computed mean scores for each load and movement direction (push, pull). When further averaged across subjects, the phase lags for the six load-by-direction combinations ranged from -3 to $21 \mathrm{msec}$ with an overall mean of $14 \mathrm{msec}$ - a positive lag indicating that GF rate led $\mathrm{LF}$ rate. A repeated measures ANOVA failed to reveal reliable effects of load $\left(F_{(2,18)}=3.04\right.$; $p=0.07)$ or direction $\left(F_{(1,9)}=0.04 ; p=0.84\right)$ on phase lag. [In contrast, the maximum correlation coefficient did vary significantly with load $\left(F_{(2,18)}=11.3 ; p=0.001\right)$ but not direction $\left(F_{(1,9)}\right.$ $=1.4 ; p=0.27)$. The mean coefficients for the inertial, viscous, and composite loads were $0.76,0.90$, and 0.76 , respectively.] Finally, no reliable correlations between phase lag and trial number were observed ( $p>0.10$ in all six loads by direction conditions). These results are consistent with the observation that GF is modulated in parallel with, and thus anticipates, fluctuations in LF.

The parallel modulation of GF and LF also is reflected in the force ratio traces shown in Figures 3-5. The force ratio provides an index of grasp stability; if the ratio drops below a critical value, 

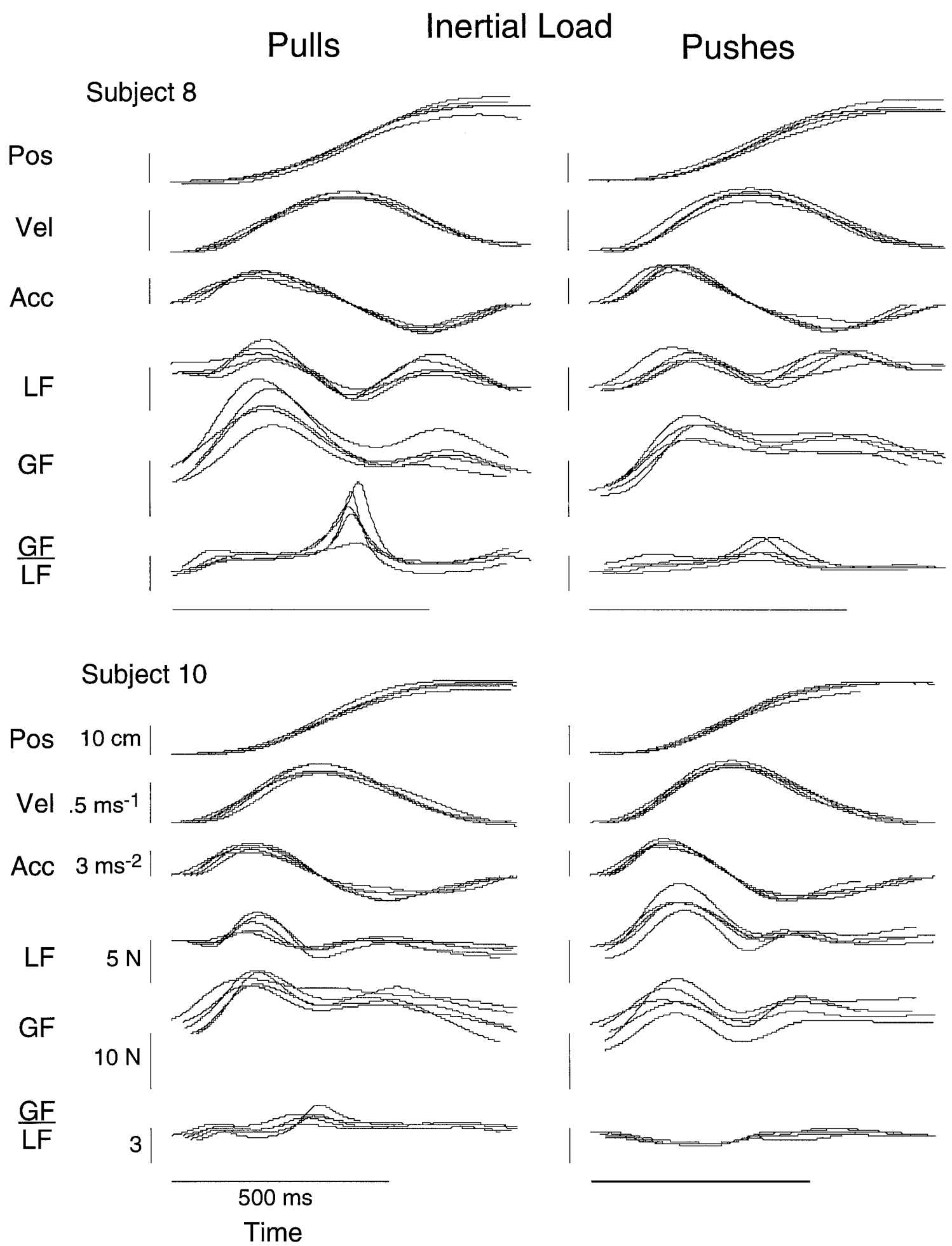

Figure 3. Overlaid kinematic and kinetic records taken from the last 20 trials under the inertial load condition (after adaptation to the load). Five push and five pull trials are shown for two subjects. Calibration values for bars are given in the bottom left panel. All bars start at zero.

slip occurs. Johansson and Westling (1984) observed that the force ratio tends toward a stable minimum value in manipulation tasks and suggested that the ratio is a controlled parameter. The minimum ratios are approximately similar across loads despite differences in the form of the force ratio curve because of differ- ences in the load function. (Note that the ratio increases rapidly when the load force approaches zero.)

To provide a summary across subjects, we computed a number of descriptive statistics on the basis of the last 30 trials (i.e., excluding the first 10 trials) from each load condition. For each 


\section{Pulls Viscous Load}
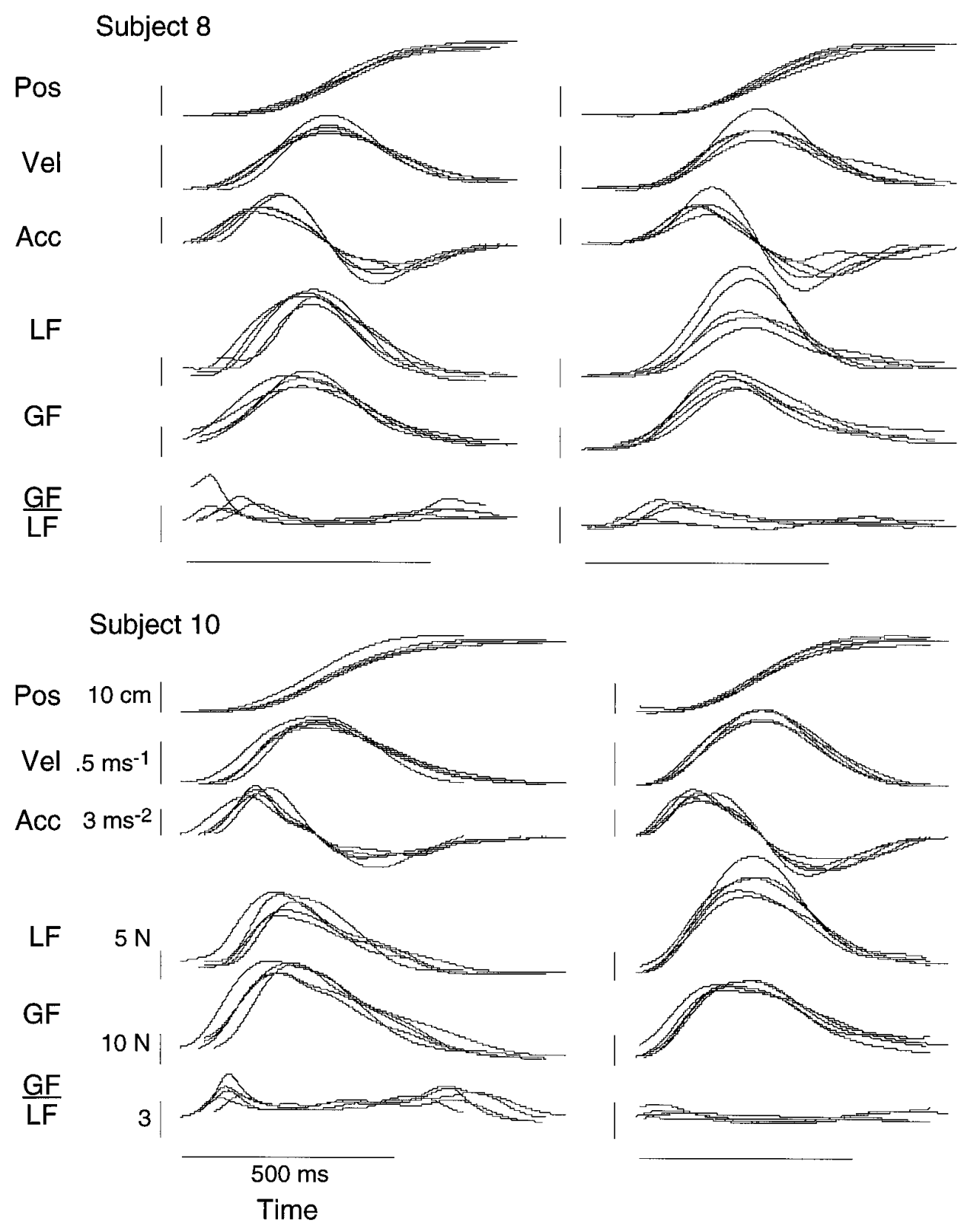

\section{Pushes}

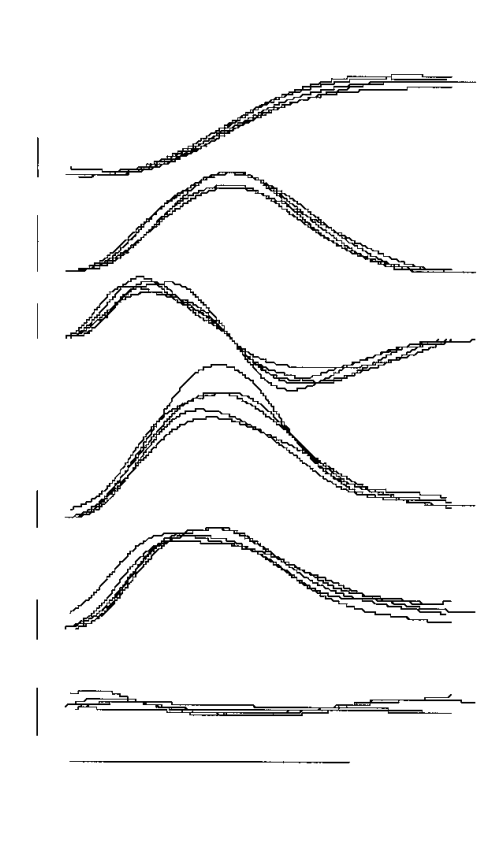

Figure 4. Overlaid kinematic and kinetic records taken from the last 20 trials under the viscous load condition (after adaptation to the load). Five push and five pull trials are shown for two subjects. Calibration values for bars are given in the bottom left panel. All bars start at zero. subject we computed a mean score for each combination of load and direction (push, pull), yielding six scores per subject. We then averaged these mean scores across subjects. Table 1 presents means and SD for the following variables: movement time (MT) and peak velocity; times to peak velocity, the initial LF peak, and the initial GF peak (all expressed as a percentage of MT); the amplitudes of the initial LF peak and the initial GF peak; and the median force ratio during the movement. Repeated measures ANOVA was used to assess the effects of load condition and movement direction on these variables.

There were significant effects of $\operatorname{load}\left(F_{(2,18)}=18.4 ; p<\right.$ $0.001)$ and direction $\left(F_{(1,9)}=5.22 ; p=0.048\right)$ on MT, but the interaction between load and direction was not reliable $\left(F_{(2,18)}\right.$ $=2.74 ; p=0.092)$. MT was less in the inertial load condition than in the other load conditions. On average, the pulls were slightly shorter than the pushes. There was a reliable effect of load on peak velocity $\left(F_{(2,18)}=17.3 ; p<0.001\right)$, but neither the effect of direction $\left(F_{(1,9)}=1.23 ; p=0.295\right)$ nor the interaction between direction and load $\left(F_{(2,18)}<1\right)$ was significant. The percentage time to peak velocity, a measure of the skewness of the velocity profile, did not vary significantly with load $\left(F_{(2,18)}\right.$ $=3.40 ; p=0.056)$ or direction $\left(F_{(1,9)}=2.04 ; p=0.187\right)$, and the interaction between load and direction $\left(F_{(2,18)}<1\right)$ was also not significant.

In the inertial load condition, the initial peaks in GF and LF both occurred approximately a quarter of the way through the movement. In the viscous load condition, the two force peaks closely coincided in time but occurred later in the movement (35-40\% of MT). Under the composite load condition, the timing of the GF and LF peaks was quite variable, often occurring at the end of the movement. The percentage time to peak GF was reliably different in the inertial and viscous load conditions $\left(F_{(1,9)}\right.$ 


\section{Pulls Elastic Load}

\section{Pushes}
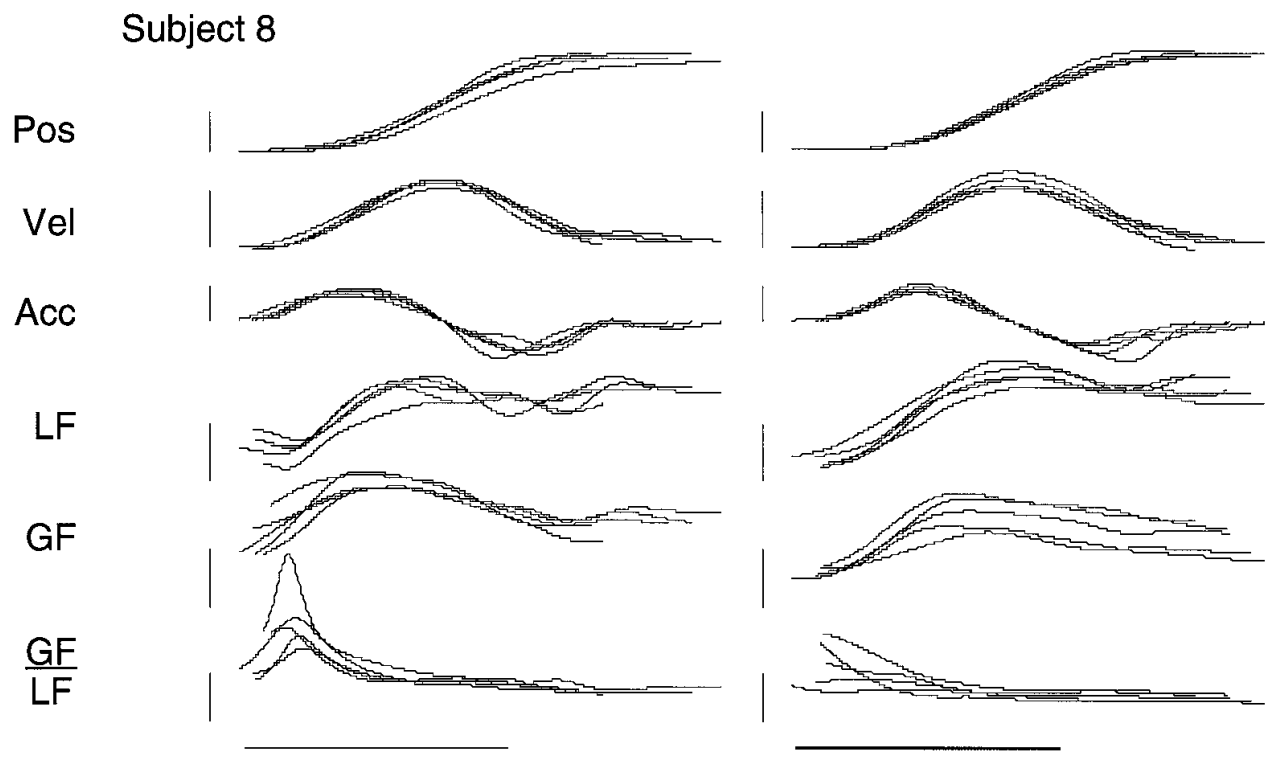

\section{Subject 10}

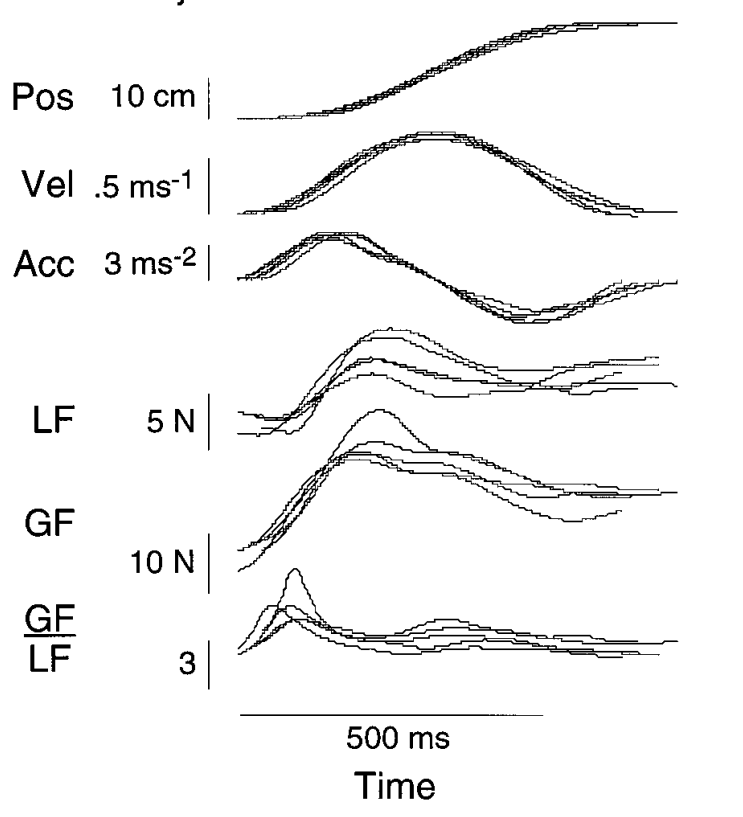

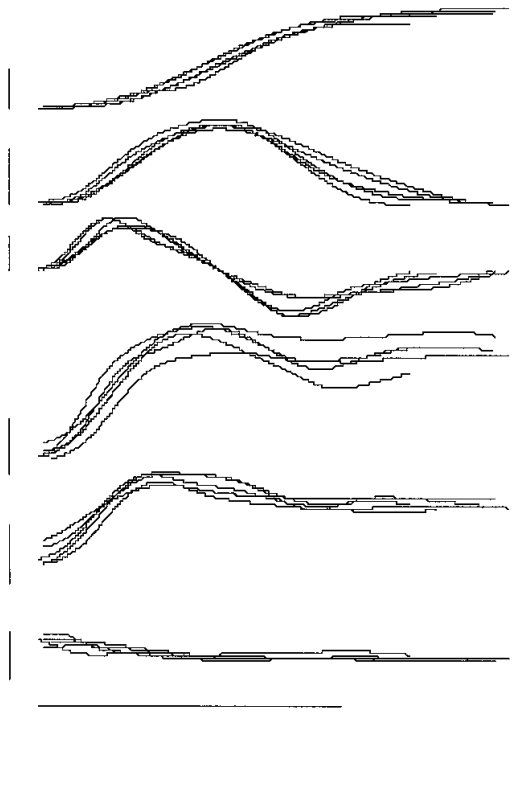

Figure 5. Overlaid kinematic and kinetic records taken from the last 20 trials under the composite load condition (after adaptation to the load). Five push and five pull trials are shown for two subjects. Calibrations for bars are given in the bottom left panel. All bars start at zero.
Separate analyses were performed for each subject and load condition (inertial, viscous), collapsing across pushes and pulls. For 18 of the 20 subject-by-load combinations, the slope of the relation between time to peak GF and time to peak LF was significant ( $r=0.38-0.93 ; p<0.05$ in all 18 cases $)$. These results indicate that, on a trial-by-trial basis, there is a close temporal coupling between GF and LF for both the inertial and viscous load conditions. To assess the extent to which the two force peaks coincided in time, we computed the time lag between peak GF and peak LF (a positive lag indicating that GF leads LF) on a trial-by-trial basis and then obtained average scores for each subject for each of the two load conditions (inertial and viscous). The mean $\pm \mathrm{SD}$ of these scores was $3 \pm$ $14 \mathrm{msec}$ for the inertial load and $19 \pm 26 \mathrm{msec}$ for the viscous load. A repeated measures ANOVA indicated that there was the first LF peak. (The composite load trials were not analyzed because of the difficulty of obtaining clearly defined peaks.)
$=218 ; p<0.001)$, as was the percentage time to peak LF $\left(F_{(1,9)}\right.$ direction $\left(F_{(1,9)}<1\right.$ in both instances).

Finally, there was a reliable effect of load condition on median force ratio $\left(F_{(2,18)}=5.1 ; p=0.018\right)$. The ratio was lowest for the viscous load and highest for the inertial load. No reliable effects were between load and direction $\left(F_{(2,18)}<1\right)$.

There is evident trial-to-trial variation in Figures 3-5, and to assess the coordination between GF and LF, we examined the relation between the time to peak GF and the time to the peak LF using linear regression analysis. For the inertial load trials, which exhibit two force peaks, we took the first GF peak and 
Table 1.

\begin{tabular}{|c|c|c|c|c|c|c|}
\hline & \multicolumn{2}{|c|}{ Inertial load } & \multicolumn{2}{|c|}{ Viscous load } & \multicolumn{2}{|c|}{ Composite load } \\
\hline & Pushes & Pulls & Pushes & Pulls & Pushes & Pulls \\
\hline \multirow[t]{2}{*}{ Movement time (MT) (sec) } & 0.72 & 0.72 & 0.82 & 0.78 & 0.87 & 0.84 \\
\hline & $(0.05)$ & $(0.06)$ & $(0.08)$ & $(0.13)$ & $(0.08)$ & $(0.09)$ \\
\hline \multirow[t]{2}{*}{ Peak velocity $(\mathrm{cm} / \mathrm{sec})$} & 71.6 & 71.4 & 68.5 & 66.7 & 56.6 & 55.6 \\
\hline & $(7.4)$ & $(8.8)$ & $(9.1)$ & $(10.3)$ & $(7.0)$ & $(8.7)$ \\
\hline \multirow[t]{2}{*}{ Time to peak velocity (\% MT) } & 46.1 & 46.9 & 42.6 & 44.0 & 45.6 & 46.2 \\
\hline & $(4.2)$ & $(3.3)$ & $(6.6)$ & $(8.3)$ & $(5.7)$ & $(4.3)$ \\
\hline \multirow[t]{2}{*}{ Peak load force (LF) (N) } & 7.1 & 7.1 & 13.4 & 13.8 & 9.79 & 9.14 \\
\hline & $(2.1)$ & $(1.2)$ & $(3.1)$ & $(2.4)$ & $(2.4)$ & $(1.1)$ \\
\hline \multirow[t]{2}{*}{ Peak grip force (GF) (N) } & 15.7 & 18.1 & 23.6 & 28.8 & 20.6 & 21.9 \\
\hline & $(5.4)$ & $(4.7)$ & $(5.7)$ & $(4.7)$ & $(3.9)$ & $(4.7)$ \\
\hline \multirow{2}{*}{ Time to peak LF (\% MT) } & 28.3 & 26.0 & 38.5 & 38.0 & & \\
\hline & $(6.3)$ & $(3.7)$ & $(6.3)$ & $(9.4)$ & & \\
\hline \multirow[t]{2}{*}{ Time to peak GF (\% MT) } & 27.3 & 26.1 & 36.6 & 35.1 & & \\
\hline & $(5.3)$ & $(4.3)$ & $(9.7)$ & (9.6) & & \\
\hline \multirow[t]{2}{*}{ Median force ratio } & 2.95 & 3.07 & 2.25 & 2.63 & 2.41 & 2.76 \\
\hline & $(1.05)$ & $(0.58)$ & $(0.46)$ & $(0.55)$ & $(0.83)$ & $(0.95)$ \\
\hline
\end{tabular}

Means and SD (in parentheses) of the mean scores of 10 subjects. Subject means were based on the last 30 trials in each load condition.

\section{Normalized Velocity Profiles for Pulls}
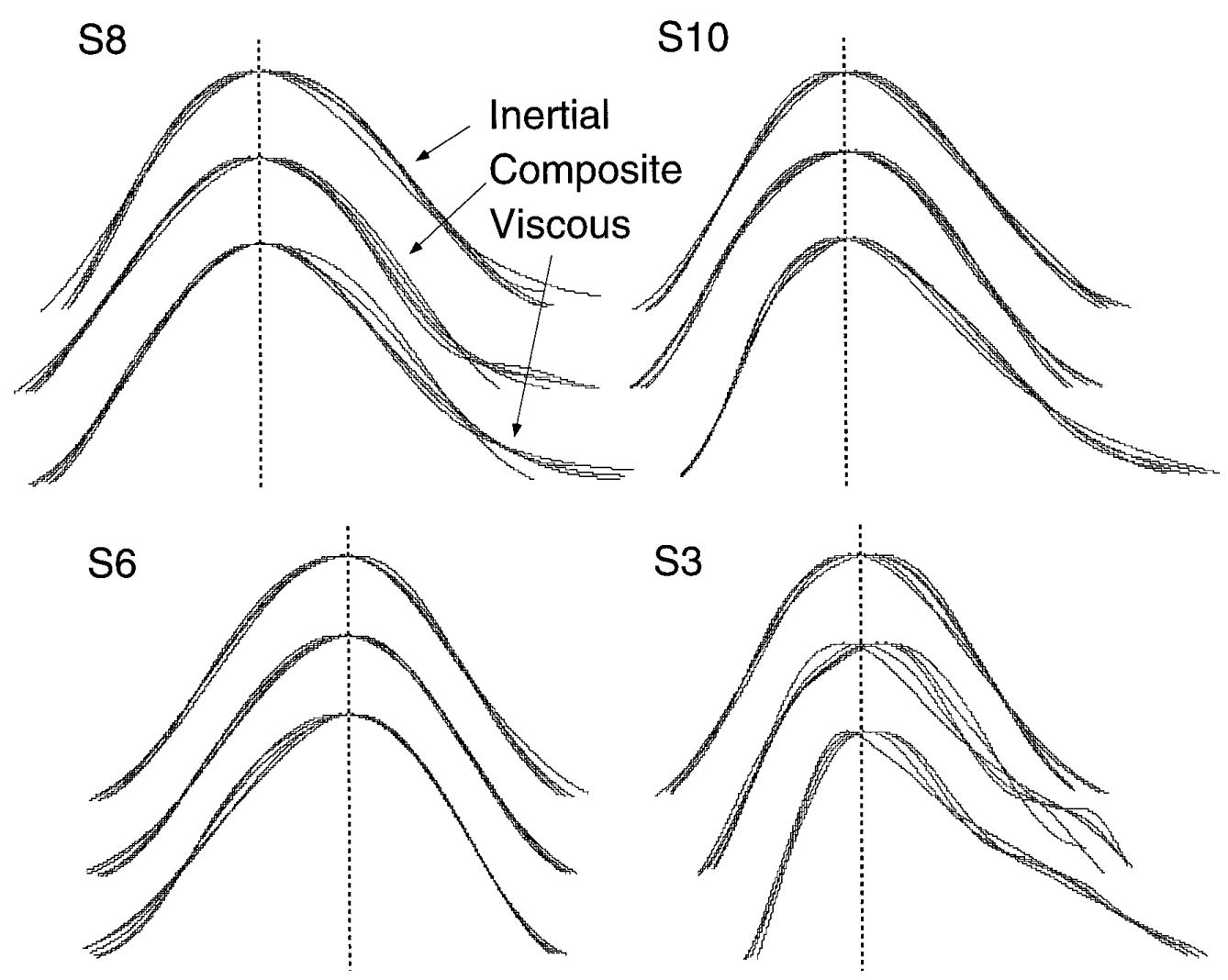

Figure 6. Five hand velocity profiles from the last 10 pull trials in each of the three load conditions for four subjects. Velocity profiles were normalized with respect to area and peak velocity and aligned with respect to peak velocity. Whereas one-half of the subjects produced profiles that were invariant across loads (illustrated by $S 8$ and $S 6$ ), the others did not (illustrated by $S 10$ and $S 3$ ).

no effect of load condition on the time lag between peak forces $\left(F_{(1,9)}=3.53 ; p=0.09\right)$. These results are in agreement with the cross-correlation phase lags reported above and indicate that GF tends to lead LF by a small margin (11 $\mathrm{msec}$ on average, overall). The findings further support the notion that modulations in GF anticipate fluctuations in LF.

\section{Hand velocity profiles}

As noted above, the form of the hand velocity profile was relatively invariant across loads for some subjects but not for others. This is illustrated in Figure 6, which shows normalized velocity profiles for four subjects taken from the last ten pull 
trials. These profiles were normalized with respect to area (i.e., displacement) and peak velocity (see Atkeson and Hollerbach, 1985 ) and then aligned to peak velocity. The figure shows data from two subjects (S6 and S8) whose velocity profiles were similar across loads and from two other subjects (S3 and S10) whose profiles under the different load conditions were visibly different. In particular, the velocity profiles under the viscous load were skewed more positively than those observed under the inertial and composite loads. This observation was confirmed quantitatively by using percentage time to peak velocity, a measure of the skew of the velocity profile. Although the overall effect of load on percentage time to peak velocity was not significant across subjects (see above), when the data from each subject were analyzed separately, differences in percentage time to peak velocity across load conditions were observed in six of the ten subjects $(p<$ 0.05 in all 6 cases). In five of these six subjects, the velocity profiles under the viscous load condition were skewed more positively than in the other two load conditions. (One subject, S7, showed the opposite result.) Thus, in one-half of the subjects, the velocity profiles were skewed more positively under viscous loading than under inertial or composite loading.

\section{DISCUSSION}

We have shown that when hand-held loads are moved that depend on different kinematic variables, GF is modulated in parallel with and thus anticipates the LF in all cases. Because LF depends on both the dynamics (i.e., load properties) of the object and the kinematics of the object, the present results indicate that the CNS (1) has an internal model of the dynamics of the object and (2) can predict the trajectory of the object. The latter prediction is, presumably, based on an internal model of the loaded limb. Thus, our results indicate that not only does the CNS build an internal model of the external load, but it also integrates the dynamics of the load into an internal model of the motor apparatus as a whole.

The finding that the CNS is able to predict precisely the different hand-held loads places strong constraints on theories of motor control. Consider, first, the inverse model of reaching, which assumes that the CNS explicitly plans the trajectory of the hand and then computes the central commands required to realize the desired trajectory. In this case, the planned trajectory of the hand could be sent to a grip force controller, which also would receive information about the properties of the object, including its dynamics and surface texture. Provided that the internal model is accurate (so that the actual kinematics match the planned kinematics), then the grip force should be adjusted appropriately. The inverse model predicts that, after load adaptation, the form of the hand trajectory will be invariant across loads. However, in onehalf of our subjects we found that the form of the hand trajectory changed as a function of load. Although several investigators have reported that the form of the hand velocity profile is unaffected by inertial (Ruitenbeek, 1984; Atkeson and Hollerbach, 1985; Bock, 1990) and velocity-dependent loads (Lackner and Dizio, 1992; Shadmehr and Mussa-Ivaldi, 1994), other workers have reported changes in the movement trajectory because of various loads (Stein et al., 1988; Uno et al., 1989). These findings speak to the flexibility of motion planning. Recently, Rosenbaum et al. (1993) have proposed a model of reaching based on the idea of weighted cost functions. According to this model, the CNS selects a trajectory by balancing various costs associated with end point accuracy, movement amplitude, etc. Within this framework, one might speculate that the two patterns of results reported here might reflect differences in cost assignment (see also Nelson, 1983).
Thus, for example, the subjects who exhibited invariance in the form of the hand velocity profile may have placed more weight on the smoothness of the trajectory than the others.

Overall, the present results are better accounted for by an internal forward model. A forward model of the dynamics of the motor apparatus and external load would enable the CNS to predict the load force acting on the hand and thus could serve as the basis of the coordination between grip force and load force. The forward model does not make any specific assumptions about the form of the hand trajectory and can be incorporated into various control schemes. For example, a forward model could be used in combination with equilibrium point (EP) control (Flash, 1987; Feldman et al., 1990; Flanagan et al., 1993a). In this case, movements would be generated by shifting the EP of the hand, and the forward model would be used to estimate the load force. Then this information would be used to control grip force, presumably by specifying the EPs of the fingertips. In addition, a high level controller might, under some conditions, modify the central commands governing the EP of the hand to produce a desired trajectory. (It is worth noting that the $\lambda$ model predicts that the form of the velocity profile will be skewed more positively under the viscous load condition than under the inertial load condition if the central commands underlying the movement are the same under both loads.)

Grip force adjustments during object transport may be considered more broadly as anticipatory postural adjustments (APAs) (Johansson and Cole, 1994; Wing, 1996). There is a large body of literature on APAs, and the focus of work in the area has been to characterize adjustments in trunk and leg muscles (measured in terms of electromyographic activity and/or ground reaction forces) that occur before arm movements (Belen'kii et al., 1967; Bouisset and Zattara, 1987). These adjustments are thought to stabilize the body in the face of potentially destabilizing reaction forces that arise during arm movement (Friedli et al., 1988). An internal model of the dynamics of the limb could be used in stabilization of the body posture in addition to stabilization of the hand-held object during arm movement (Flanagan et al., 1995; Wing et al., 1997).

Given the evidence favoring the existence of an internal model, the question naturally arises as to the neural mechanisms involved. Miall et al. (1993) have suggested that the cerebellum makes use of two internal models: a forward model of the motor apparatus, which provides a rapid prediction of the sensory consequences of motor commands, and a second model of the time lags in the control loop because of receptor and effector delays, conduction times, and so on. The second model delays the predicted sensory feedback so that it can be compared directly with the actual sensory feedback. The error signal from this comparison may be used to modify motor commands during performance and to update the first model. In contrast, Kawato and Gomi (1992) suggested that the cerebellum functions as an inverse model to translate information about the desired trajectory, provided by signals from parietal cortex, into the required motor commands. In line with this idea, Kalaska (1991) has proposed that superior parietal cortex may provide a neuronal representation of kinematics for kinesthetic perception as well as for movement control.

The models discussed above confer a critical role to sensory feedback both in modifying the ongoing movement and in parameterizing the internal model (see also Ghez et al., 1991). In this regard it is interesting to note that patients with peripheral sensory neuropathy produce arm movements that are uncoordinated 
because of failure to allow for intersegmental dynamics (Sainburg et al., 1993). Thus, although the cerebellum may provide predictive control, it nevertheless depends on sensory feedback about current movement conditions. Sainburg et al. observed that vision markedly improved performance in deafferented patients. Thus, it seems likely that the patient's deficits were attributable to a lack of information, such as initial limb position, required by the cerebellar model rather than a failure of the internal model itself. However, the results also suggest that the default internal model, used in the absence of vision, is not accurate.

Support for the suggestion that the cerebellum contributes to anticipatory GF adjustments comes from a study by Müller and Dichgans (1994). These authors reported that patients with degenerative cerebellar lesions exhibited a lack of coordination of GF and LF when performing a lifting task and using a precision grip. In these patients, GF and LF were decoupled somewhat such that the two forces did not always change in parallel. In contrast, when normal controls perform this task, GF and LF are modulated in parallel (Johansson and Westling, 1984). Moreover, over trials, the cerebellar patients did not adapt their GF rise rates to match different loads. Although the patients were able to adjust grip force rates to some degree, they did so significantly less efficiently than control subjects. Müller and Dichgans (1994) concluded that this represented a failure of anticipatory parameterization. It is interesting to contrast the performance of cerebellar patients with that of parkinsonian subjects who can appropriately scale their grip force for different weights when lifting, although they use high grip force levels (Müller and Abbs, 1990). The deficit in Parkinson's disease may be associated with problems with force production rather than anticipatory control. Neurophysiological evidence that the cerebellum contributes to anticipatory GF adjustments comes from the work of Smith and colleagues. These researchers have reported that the discharge of neurons in cerebellum (Espinosa and Smith, 1990), as well as primary motor cortex (Picard and Smith, 1992), is related to object weight and texture before movement onset.

The idea that anticipatory GF adjustments are based on an internal model of the motor system fits well within the more general model of precision manipulation proposed by Johansson and colleagues, referred to as "discrete event, sensory-driven control" (Johansson and Cole, 1992, 1994; Johansson and Edin, 1993). According to this control scheme, precision manipulation involves subtle interplay between feedforward and feedback mechanisms. Feedforward control, based on an internal model of the motor system and memory of object properties, is used to specify motor commands in advance of the movement. During the movement, sensory feedback from cutaneous mechanoreceptors and other sources is used intermittently to inform the CNS about the completion of various phases of the task and to trigger subsequent phases. For example, during a task in which an object is lifted from a support surface with a precision grip and then replaced, fast-adapting type I (FA I) afferents reliably signal the initial contact and final release of the digits, and FA II afferents (Pacinian corpuscles) are extremely sensitive to the mechanical transients associated with the lift-off and touch-down of the object (Westling and Johansson, 1987). In addition, sensory feedback is used to update inappropriate motor commands that lead to mechanical events, such as slip or the generation of excess grip forces. Also, this information presumably is used to update the internal model of the motor apparatus and object (Johansson and Westling, 1987; Johansson, 1996).

\section{REFERENCES}

Atkeson CG (1989) Learning arm kinematics and dynamics. Annu Rev Neurosci 12:157-183.

Atkeson CG, Hollerbach JM (1985) Kinematic feature of unrestrained vertical arm movements. J Neurosci 5:2318-2330.

Belen'kii VE, Gurfinkel VS, Pal'tsev EI (1967) On the elements of control of voluntary movement. Biofizika 12:135-141.

Bizzi E, Accornero N, Chapple W, Hogan N (1984) Posture control and trajectory formation during arm movement. J Neurosci 4:2738-2744.

Bouisset S, Zattara M (1987) Biomechanical study of the programming of anticipatory postural adjustments associated with voluntary movement. J Biomech 20:735-742.

Bock O (1990) Load compensation in human goal-directed arm movements. Behav Brain Res 41:167-177.

Bullock D, Grossberg S (1988) Neural dynamics of planned arm movements: emergent invariants and speed-accuracy properties during trajectory formation. Psychol Rev 95:49-90.

Espinosa E, Smith AM (1990) Purkinje cell simple spike activity during grasping and lifting objects of different textures and weights. J Neurophysiol 64:698-714.

Feldman AG, Adamovich SV, Ostry DJ, Flanagan JR (1990) The origins of electromyograms-explanations based on the equilibrium point hypothesis. In: Multiple muscle systems: biomechanics and movement organization (Winters J, Woo S, eds), pp 195-213. London: Springer.

Flanagan JR, Tresilian JR (1994) Grip-load force coupling: a general control strategy for transporting objects. J Exp Psychol Hum Percept Perform 20:944-957.

Flanagan JR, Wing AM (1993) Modulation of grip force with load force during point-to-point movements. Exp Brain Res 95:131-143.

Flanagan JR, Wing AM (1995) The stability of precision grip force during cyclic arm movements with a hand-held load. Exp Brain Res 105:455-464.

Flanagan JR, Ostry DJ, Feldman AG (1993a) Control of trajectory modifications in reaching. J Mot Behav 25:140-152.

Flanagan JR, Tresilian JR, Wing AM (1993b) Coupling of grip force and load force during arm movements with grasped objects. Neurosci Lett 152:53-56.

Flanagan JR, Tresilian JR, Wing AM (1995) Grip force adjustments during rapid hand movements suggest that detailed movement kinematics are predicted. Behav Brain Sci 18:753-754.

Flash T (1987) The control of hand equilibrium trajectories in multi-joint arm movements. Biol Cybern 57:57-74.

Friedli WG, Cohen L, Hallett M, Stanhope S, Simon SR (1988) Postural adjustments associated with rapid arm movements. II. Biomechanical analysis. J Neurol Neurosurg Psychiatry 51:232-243.

Ghez C, Henning W, Gordon J (1991) Organization of voluntary movement. Curr Opin Neurobiol 1:664-671.

Hollerbach JM (1990) Planning of arm movements. In: Visual cognition and action, Vol. 2 (Osherson DN, Kosslyn SM, Hollerbach JM, eds), pp 183-211. Cambridge, MA: MIT.

Johansson RS (1996) Sensory control of dextrous manipulation in humans. In: Hand and brain: neurophysiology and psychology of hand movement (Wing AM, Haggard P, Flanagan JR, eds), pp 381-414. San Diego: Academic.

Johansson RS, Cole KJ (1992) Sensory-motor coordination during grasping and manipulation actions. Curr Opin Neurobiol 2:815-823.

Johansson RS, Cole KJ (1994) Grasp stability during manipulative actions. Can J Physiol Pharmacol 72:511-524.

Johansson RS, Edin BB (1993) Predictive feedforward sensory control during grasping and manipulation in man. Biomed Res 14:95-106.

Johansson RS, Westling G (1984) Roles of glabrous skin receptors and sensorimotor memory in automatic control of precision grip when lifting rougher or more slippery objects. Exp Brain Res 56:550-564.

Johansson RS, Westling G (1987) Signals in tactile afferents from the fingers eliciting adaptive motor responses during precision grip. Exp Brain Res 66:141-154.

Johansson RS, Riso R, Hager C, Backstrom L (1992) Somatosensory control of precision grip during unpredictable pulling loads. Exp Brain Res 89:181-191.

Jordan MI, Rumelhart DE (1992) Forward models: supervised learning with a distal teacher. Cognit Sci 16:307-354.

Jordan MI, Flash T, Arnon Y (1994) A model of the learning of arm trajectories from spatial deviations. J Cognit Neurosci 6:359-376.

Kalaska JF (1991) Parietal cortex area 5: a neuronal representation of move- 
ment kinematics for kinaesthetic perception and movement control. In: Brain and space (Paillard J, ed), pp 133-146. Oxford: Oxford UP.

Kawato M, Gomi H (1992) A computational model of four regions of the cerebellum based on feedback-error learning. Biol Cybern 68:95-103.

Lackner JR, Dizio P (1992) Rapid adaptation of arm movement endpoint and trajectory to Coriolis force perturbations. Soc Neurosci Abstr 22:515.

Miall RC, Weir DJ, Wolpert DM, Stein JF (1993) Is the cerebellum a Smith predictor? J Mot Behav 25:203-216.

Müller F, Abbs JH (1990) Precision grip in parkinsonian patients. In: Advances in neurology (Streifer MB, Korezyn AD, Melamed E, You$\operatorname{dim}$ MBH, eds), pp 191-195. New York: Raven.

Müller F, Dichgans J (1994) Dyscoordination of pinch and lift forces during grasp in patients with cerebellar lesions. Exp Brain Res 101:485-492.

Nelson WL (1983) Physical principles for economies of skilled movements. Biol Cybern 46:135-147.

Picard N, Smith AM (1992) Primary motor cortical activity related to weight and texture of a grasped object. J Neurophysiol 68:1867-1881.

Rosenbaum DA, Engelbrecht SE, Bushe MM, Loukopoulos LD (1993) Knowledge model of selecting and producing reaching movements. J Mot Behav 25:217-227.
Ruitenbeek JC (1984) Invariants in loaded goal-directed movements. Biol Cybern 51:11-20.

Sainburg RL, Poizner H, Ghez C (1993) Loss of proprioception produces deficits in interjoint coordination. J Neurophysiol 70:2136-2147.

Saltzman E (1979) Levels of sensorimotor representation. J Math Psychol 20:91-163.

Shadmehr R, Mussa-Ivaldi FA (1994) Adaptive representation of dynamics during learning of a motor task. J Neurosci 14:3208-3224.

Stein RB, Cody FWJ, Capaday C (1988) The trajectory of human wrist movements. J Neurophysiol 59:1814-1830.

Uno Y, Kawato M, Suzuki R (1989) Formation and control of optimal trajectory in human arm movement. Biol Cybern 61:89-101.

Westling G, Johansson RS (1987) Factors influencing the force control during precision grip. Exp Brain Res 53:277-284.

Wing AM (1996) Anticipatory control of grip force in rapid arm movements. In: Hand and brain: neurophysiology and psychology of hand movement (Wing AM, Haggard P, Flanagan JR, eds), pp 301-324. San Diego: Academic.

Wing AM, Flanagan JR, Richardson J (1997) Anticipatory postural adjustments in stance and grip. Exp Brain Res, in press.

Wolpert DM, Ghahramani Z, Jordan MI (1995) An internal model for sensorimotor integration. Science 269:1880-1882. 